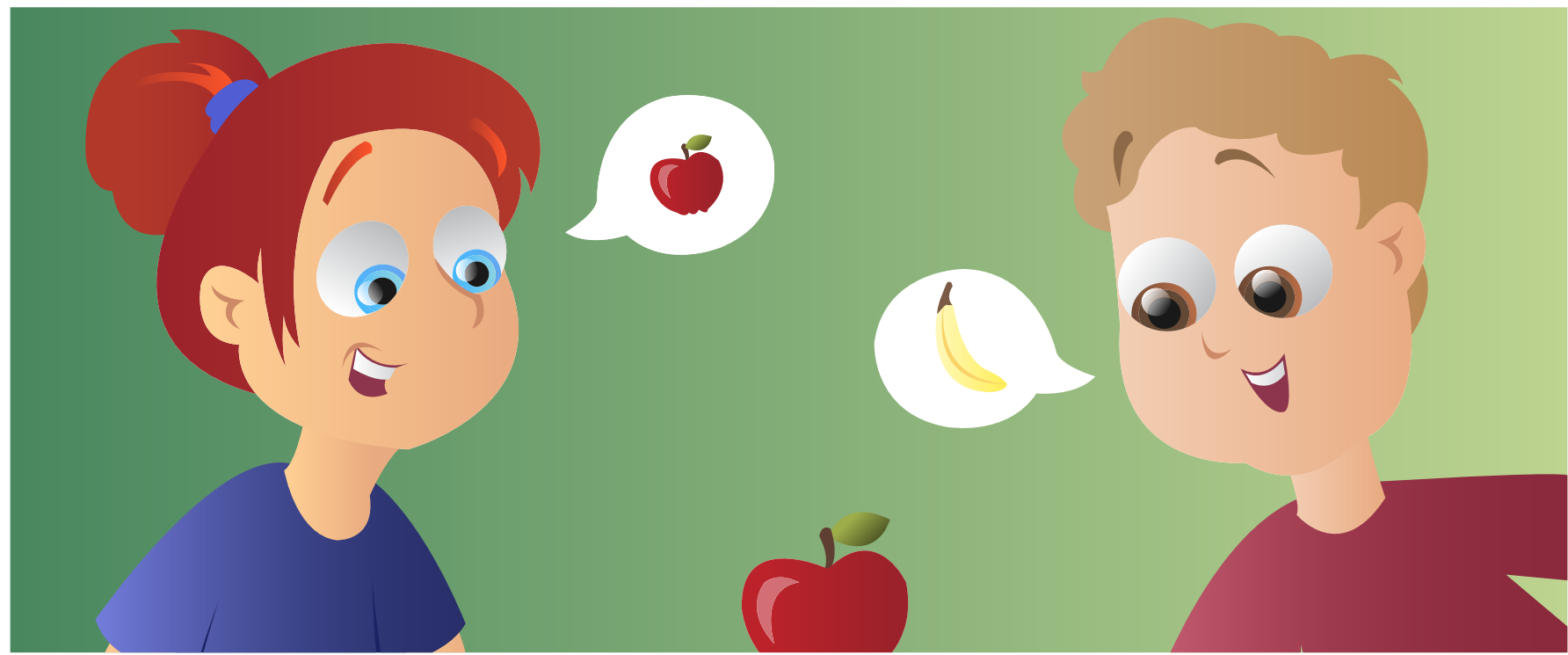

\title{
KNOWING WHAT WE SEE
}

Chris B. Martin ${ }^{1 *}$, Celia Fidalgo ${ }^{1}$, and Morgan D. Barense ${ }^{1,2}$

${ }^{1}$ Department of Psychology, University of Toronto, Toronto, ON, Canada, ${ }^{2}$ Rotman Research Institute, Toronto, ON, Canada

\section{REVIEWED BY:}

PRINCETON

FRIENDS

$\mathrm{SCHOOL}$

11-12 YEARS OLD
Have you ever wondered how you know what you see? For example, when you see a round, red object at the grocery store, how do you know that it is called an apple and that it can be eaten? This type of knowledge is called semantic memory. Semantic memories are learned over a lifetime and can be accessed without remembering a specific experience. In other words, you do not need to remember details from the last time you ate an apple to identify one at the grocery store. Although knowledge about apples seems to come to mind rather easily when we see them, linking semantic memory to vision actually requires a lot of brain power! The purpose of this paper is to explain how the brain accomplishes this goal and to describe the functions of key brain regions. We also discuss the functional importance of linking semantic memory and vision.

\section{KNOWING WHAT WE SEE}

If asked to describe the environment that you are in right now, you would likely begin by naming everything you see, such as a desk, a computer, an apple, and a banana. This might seem easy, but have you ever wondered how you know what you see? For example, how do you know that the round, red object on your desk is an apple and that it can be eaten? How do you know that apples 
VENTRAL VISUAL PATHWAY

A network of brain regions that connects areas responsible for the processing of visual information with those responsible for semantic memory, episodic memory, learning, and emotion.

\section{FIGURE}

The ventral visual pathway.

A. This panel shows where the primary visual cortex (green) and the anterior temporal lobes (blue) are located in the human brain. The arrow connecting these shaded regions is the ventral visual pathway. B. This panel shows how visual information is combined as it progresses forward through the ventral visual pathway. Simple visual features are located in the back of the brain. Invariant objects and semantic memory are represented in the anterior temporal lobe. and bananas are both fruits, even though they look very different? Although vision begins with the eyes, your brain is responsible for linking visual information to knowledge about objects. In this paper, we will describe why this process is important and how it occurs in the brain.

\section{THE VENTRAL VISUAL PATHWAY}

The first step toward knowing what you see occurs when visual information is sent from the eyes to the brain. The primary visual cortex, which is located at the back of the brain, is one of the first brain regions to receive information from the eyes. This region processes only the most basic information about the visual world, such as lines, edges, color, and direction of motion. The primary visual cortex then sends this basic information forward through the brain along the ventral visual pathway (ventral is from Latin venter, meaning "belly"; see Figure 1). The information processing performed by the ventral visual pathway supports our conscious awareness of what we are seeing, which allows us to talk about what we see.

The ventral visual pathway works to combine simple visual information, like lines, edges, and color, to create entire objects. Information is sent forward from one brain region to another in a series of steps. First, lines and edges are passed from the primary visual cortex to another brain region that combines lines and edges to create a simple shape, like a triangle or an oval. This shape is passed forward to another brain area, which fills in the shape with colors. Another area adds textures to the shape. This process continues so that, along the ventral visual pathway, objects become more complex. Some examples of complex objects might be a nose, eyes, and a mouth, or wheels, a seat, a frame, and handlebars. Further forward along the pathway, a separate brain region combines all the features of the object together, creating an entire object, such

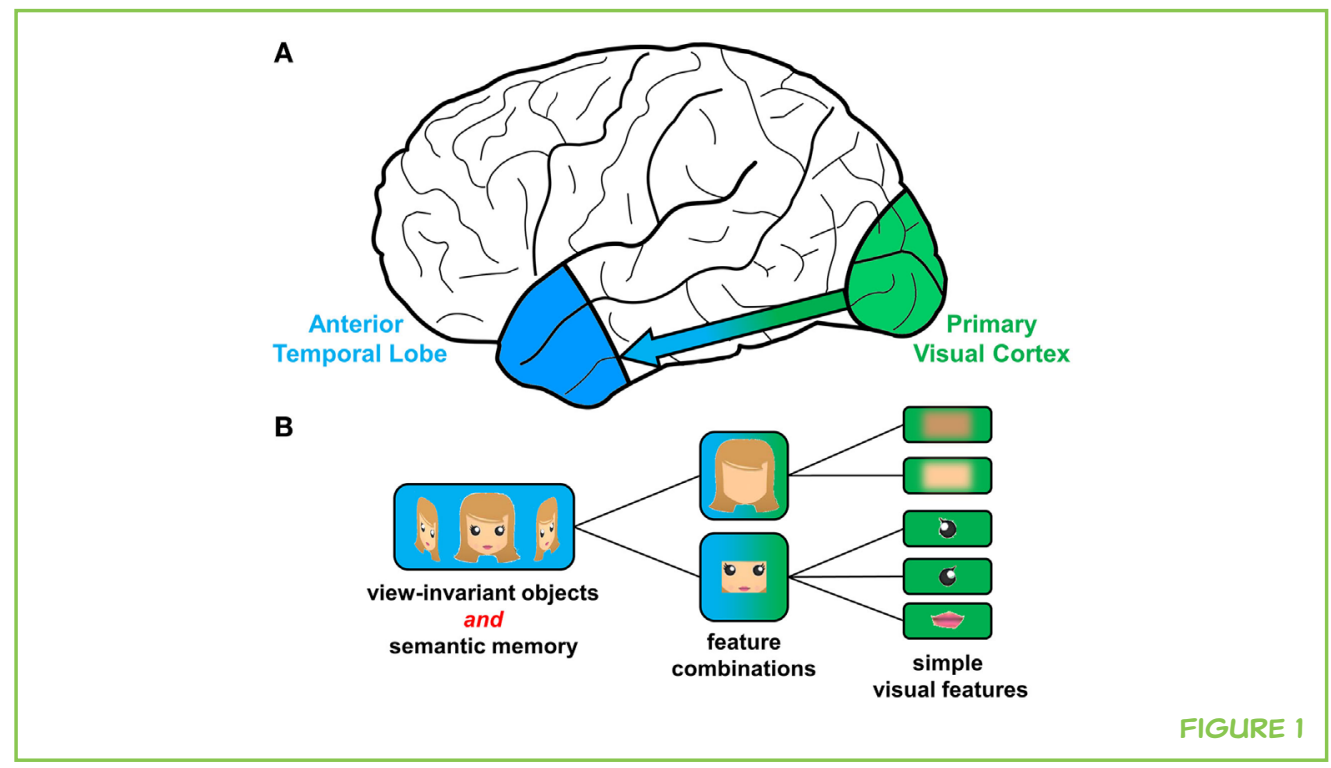




\section{SEMANTIC MEMORY}

The ability to learn and remember knowledge and facts about the world.

Semantic memories are learned slowly over time. Knowing that the Statue of Liberty was a gift from the people of France to the people of the United States is an example of an episodic memory.

\section{EPISODIC MEMORY}

The ability to store and remember information about specific events that you have personally experienced.

Remembering the first time that you saw the Statue of Liberty is an example of an episodic memory.

\section{FIGURE 2}

Semantic and episodic memory.

Semantic memory, on the left, refers to knowledge that is learned over many experiences. The contents of semantic memory can be brought to mind without remembering details from a specific event. Episodic memory, on the right, refers to memory for specific events, such as the time you picked apples at an orchard with your family. as a face or a bicycle. At the last stage in the pathway, a brain area called the anterior temporal lobe codes objects in an invariant manner [1]. The word "invariant" means that the brain represents objects in a way that does not change ("in-," which means "does not," and "variant," which means "change"). In vision, the word invariant means that we are able to see an object from different angles, distances, and lighting conditions, and still know it is the same object, despite differences in the way it looks. For example, you need to see your school teacher in an invariant way so that you do not think she is a completely different person when she turns her head away from you to look at another student on the other side of the classroom! The same idea is true of everyday objects, too. For example, you need to be able to recognize your bicycle regardless of whether it is being ridden by a friend, is leaning upright against a wall, or is lying sideways on the ground. The fact that the brain can represent objects in an invariant way is really important. It means that the objects we see can be connected to other types of information in the brain, such as general object knowledge, which we call semantic memory.

\section{WHAT IS SEMANTIC MEMORY?}

In general, memory is the ability to hold information in your brain over a period of time without being reminded. There are different kinds of memory depending on the type of information or the amount of time that it is held. In general, when people talk about memory in daily life they are referring to what is called declarative memory, which is the ability to consciously remember something from the past. There are two types of declarative memory: episodic memory and semantic memory (see Figure 2). Episodic memory is the ability to store and remember information about specific events that you have personally experienced. For example, remembering that you picked apples at an orchard with your family last summer is an episodic memory.

Semantic memory is quite different from episodic memory. Instead of memory for the things that have happened in your life, semantic memory refers to knowledge and facts about the world that are stored in your brain. Your episodic memories belong only to you-other people do not have your

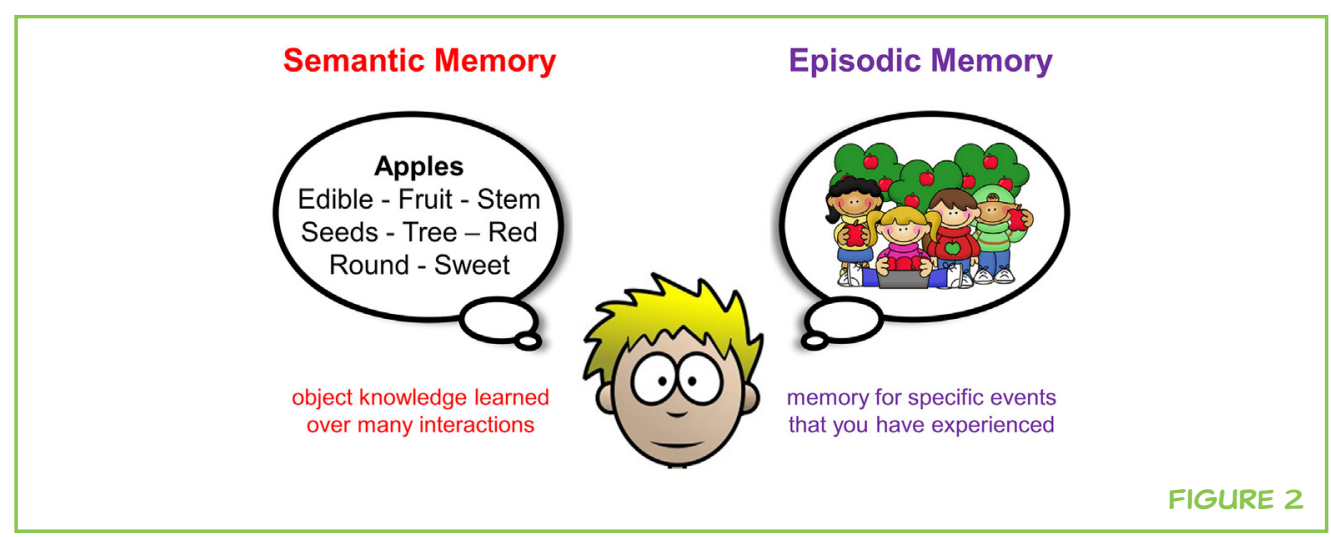




\section{SEMANTIC}

\section{DEMENTIA}

The gradual loss of semantic memories, including names of objects, their features, and how objects, are related to one another. Semantic dementia is caused by damage to the anterior temporal lobes.

\section{FIGURE 3}

Delayed-copy drawing in semantic dementia.

This figure shows the loss of object knowledge in a person with semantic dementia. The person first looked at a picture of an animal (top row) and was then asked to draw it after a delay of just $10 \mathrm{~s}$. As can be seen in the bottom row, the drawings lack detail and look more like each other than they do the actual animals the participants were asked to draw. Adapted by permission from Macmillan Publishers Ltd., Nature Reviews Neuroscience, Patterson et al. [2], Copyright 2007. memories. However, your semantic memories are shared with other people and have been learned slowly over time. In other words, you do not need to remember a specific experience with an apple to know that it is an edible fruit. This knowledge about apples is an example of a semantic memory. But being an "edible fruit" does not only describe apples-it also describes bananas, pears, and lots of other food we eat. Therefore, the concept of an apple is stored in semantic memory as a collection of features. The collection of features that only describes an apple includes that they are round, red or green, sweet, have a stem, have seeds, and grow on trees. Because object knowledge is stored as collections of features, we can quickly understand how objects relate to one another. For example, we know that apples and bananas are similar because they share a number of features-they are fruits, edible, sweet, and grow on trees.

\section{SEMANTIC DEMENTIA-WHEN SALLY THE CAMEL HAS NO HUMPS}

In psychology (the study of the brain and mind), one of the most interesting ways to understand how the brain works is to examine what happens when it is damaged. In fact, much of what we know about semantic memory comes from people who have a condition called semantic dementia, which causes semantic memory problems. People with semantic dementia have a specific kind of brain damage that causes them to lose knowledge about everyday objects, including the names of the objects, their features, and how objects are related to one another. For example, when shown a picture of a camel, a person with semantic dementia might not know why it has a hump on its back, or where it lives, and mistakenly call it a dog. As their disease gets worse, people with semantic dementia might even forget the names of all animals, and only be able to say that a picture is an "animal" without knowing what kind of animal it is. This loss of object knowledge can clearly be seen in the drawings made by people with semantic dementia [2]. As can be seen in Figure 3, when asked to draw a duck, camel, seal, and frog from memory, the drawings they create look highly similar even though these animals actually look quite different-all of the drawings include features that are shared across most animals, such as a body, head, four legs, and tail. Importantly, people with

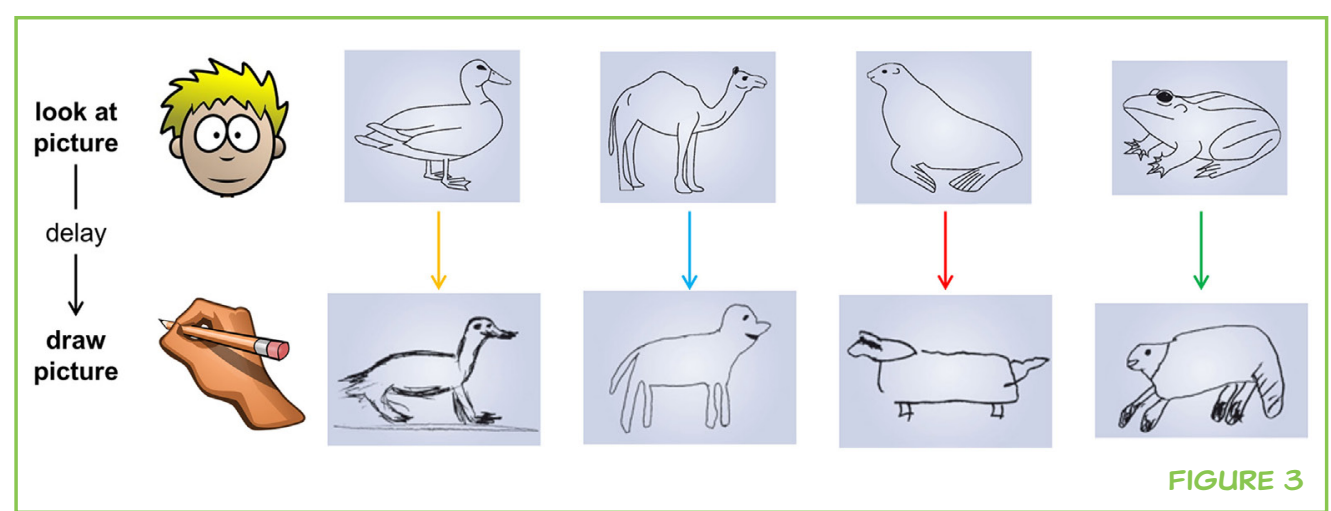


semantic dementia do not draw any features that uniquely describe any of the animals, such as the camel's hump or the seal's flippers. These are not the only mistakes semantic dementia patients make. Sometimes they may hold or move an object the wrong way [3]. For example, a patient with semantic dementia might call a toothbrush a spoon, hold it by the scoop end, and try to use it like a screwdriver.

\section{WHERE IS SEMANTIC MEMORY STORED IN THE BRAIN?}

One goal in neuroscience is to understand how different areas of the brain support different types of memories. One way to accomplish this goal is to examine the brains of people who have conditions that affect specific types of memory. For example, if we want to understand where in the brain semantic memory is stored, we can look at the brains of semantic dementia patients who have problems remembering information about objects. If a group of patients with semantic dementia all have damage to the same brain region, then we can conclude that this brain region plays an important role in semantic memory. Luckily, we have machines that allow us to do exactly this. Magnetic resonance imaging (sometimes shortened to MRI) uses powerful magnetic fields to take pictures of the brain. When we look at these pictures, we can see where a patient has brain damage. This technique has shown that the brain's anterior temporal lobes are often damaged in patients with semantic dementia. This suggests that semantic memory, including object knowledge, is stored in the anterior temporal lobes. As discussed earlier, the anterior temporal lobes are also the region in which objects are represented in an invariant manner within the ventral visual pathway (see Figure 1). These findings indicate that invariant object representations are linked to semantic memory in the anterior temporal lobes.

\section{WHAT IS THE PURPOSE OF SEMANTIC MEMORY?}

As a final point, it is worth asking what semantic memory is good for and why it evolved in the first place. To answer these questions, we need to return to vision! Humans, and many other species, rely on vision to guide how they interact with the world. For example, when we walk, we use vision to make sure that we do not bump into or trip over obstacles in our path. We also use vision to carefully guide our hand while cutting an apple with a sharp knife. However, when you first see an object, how does your brain know what to do with it? For example, when you reach into a drawer to grab a knife, how do you know that you should hold the handle rather than the blade? Semantic memory plays an important role in these decisions. Knowledge about how an object works is an important part of the collection of features stored in semantic memory. In other words, semantic memory includes knowledge about when and how we use different types of objects. As an example, "handle" is a feature 
of knives, and based on prior experience, we know that we hold objects by their handle, not by their blade!

Patients with semantic dementia show us how semantic memory influences the way we interact with the world. As discussed earlier, these patients have damage to the anterior temporal lobes of the brain. Because of this, they have forgotten much of their object knowledge. Patients with semantic dementia are able to pick up objects, but they might not know what the object is used for. For example, they might have forgotten that the bristles at the end of a toothbrush are used for brushing teeth, so they might hold a toothbrush by its brush end. Interestingly, in some situations, healthy people can even be tricked into making these types of mistakes. Normally, when healthy people are asked to pick up objects from a table, they will do so by grabbing the handles, even if the handle is facing away from them. However, when people are asked to grab objects while they also-at the same time-do another distracting semantic memory task, such as naming the capital of France, they often grab the objects by whichever end is closest to them, just like patients with semantic dementia [4]. In other words, when we are distracted, we might grab the brush end of a toothbrush instead of the handle. These errors happen because the semantic memory system is busy solving the distracting semantic memory task and is unable to recall "function" knowledge about the way objects are supposed to be picked up.

\section{SUMMARY}

In summary, we know what we see because of the way the brain processes visual information in the ventral visual pathway. Everything we see is first processed by our eyes. Our eyes then send this information to the primary visual cortex at the back of the brain. From here, simple features of the world (lines, edges, and color) are combined in steps along the ventral visual pathway. For example, in one step, lines are combined to make shapes, then in another step, shape is combined with color. The final step of the ventral visual pathway is in the anterior temporal lobes. Here, all of the visual information is combined to create entire objects. The anterior temporal lobes then link the objects we are seeing to what we know about them, which is semantic memory. Importantly, semantic memory tells us how to use objects by providing knowledge about their function.

\section{REFERENCES}

1. Mishkin, M., Ungerleider, L. G., and Macko, K. A. 1983. Object vision and spatial vision: two cortical pathways. Trends Neurosci. 6:414-7. doi:10.1016/01662236(83)90190-X

2. Patterson, K., Nestor, P. J., and Rogers, T. T. 2007. Where do you know what you know? The representation of semantic knowledge in the human brain. Nat. Rev. Neurosci. 8(12):976-87. doi:10.1038/nrn2277 
3. Hodges, J. R., Bozeat, S., Ralph, M. A. L., Patterson, K., and Spatt, J. 2000. The role of conceptual knowledge in object use evidence from semantic dementia. Brain 123(9):1913-25. doi:10.1093/brain/123.9.1913

4. Creem, S. H., and Proffitt, D. R. 2001. Grasping objects by their handles: a necessary interaction between cognition and action. J. Exp. Psychol. Hum. Percept. Perform. 27(1):218. doi:10.1037/0096-1523.27.1.218

SUBMITTED: 20 July 2016; ACCEPTED: 13 April 2017; PUBLISHED ONLINE: 10 May 2017.

EDITED BY: Robert T. Knight, University of California, Berkeley, USA

CITATION: Martin CB, Fidalgo C and Barense MD (2017) Knowing What We See. Front. Young Minds 5:15. doi:10.3389/frym.2017.00015

CONFLICT OF INTEREST STATEMENT: The authors declare that the research was conducted in the absence of any commercial or financial relationships that could be construed as a potential conflict of interest.

COPYRIGHT () 2017 Martin, Fidalgo and Barense. This is an open-access article distributed under the terms of the Creative Commons Attribution License (CC BY). The use, distribution or reproduction in other forums is permitted, provided the original author(s) or licensor are credited and that the original publication in this journal is cited, in accordance with accepted academic practice. No use, distribution or reproduction is permitted which does not comply with these terms.

\section{REVIEWED BY}

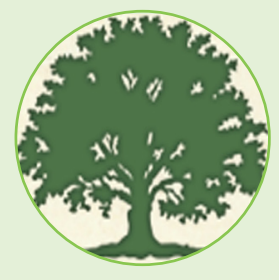

\section{PRINCETON FRIENDS SCHOOL, 11-12 YEARS OLD}

The sixth and seventh grade students at Princeton Friends School are a bright group of scientists whose interests span several scientific fields. This is an exploratory group that likes to interact with science and form ideas by doing. Through the brain discussions and review, the students are thinking more deeply about what they observe, how they process, and what drives their reactions.

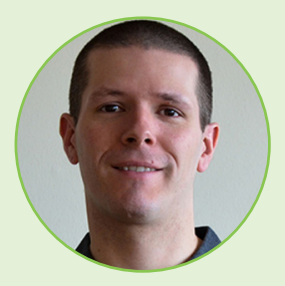

\section{AUTHORS}

\section{CHRIS B. MARTIN}

I am a postdoctoral fellow at the University of Toronto. Whether we realize it or not, memory plays an important role in nearly every aspect of our lives. For example, remembering a family vacation, riding a bike, and even your sense of personal identity is dependent on memory. It is perhaps not surprising, then, that a key goal of neuroscience research is to better understand how the brain supports memory. As a scientist, I am fortunate to be able to help uncover (some of) the answers!. *cmarti97@gmail.com 


\section{CELIA FIDALGO}

I am a graduate student studying how the brain creates memories. I think it is fascinating that the brain has such a large capacity to remember all the different objects and different places we encounter in the world. In my research, I try to understand why memories are forgotten and how this happens in the brain.

\section{MORGAN D. BARENSE}

I am a professor at the University of Toronto. I want to understand how our brains allow us to create memories. I believe that if we can better understand how the brain works, we will be able to better treat people who have brain diseases, like Alzheimer's disease or semantic dementia. I love discovering new things and figuring out how complicated processes fit together, which means I am very happy being a scientist! 